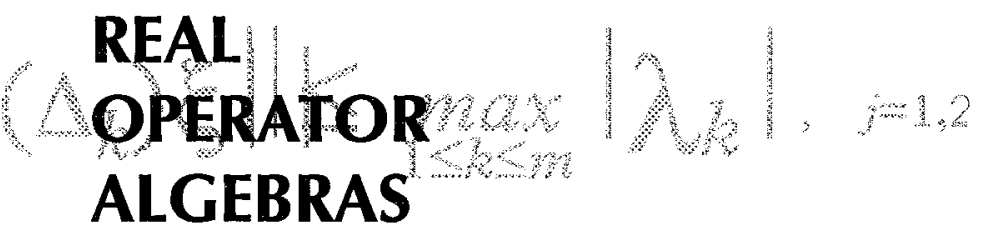


This page is intentionally left blank 


\section{REAL OPERATOR ALGEBRAS}




\section{Published by}

World Scientific Publishing Co. Pte. Ltd.

5 Toh Tuck Link, Singapore 596224

USA office: Suite 202, 1060 Main Street, River Edge, NJ 07661

UK office: 57 Shelton Street, Covent Garden, London WC2H 9HE

\section{British Library Cataloguing-in-Publication Data}

A catalogue record for this book is available from the British Library.

\section{REAL OPERATOR ALGEBRAS}

Copyright $\odot 2003$ by World Scientific Publishing Co. Pte. Ltd.

All rights reserved. This book, or parts thereof, may not be reproduced in any form or by any means, electronic or mechanical, including photocopying, recording or any information storage and retrieval system now known or to be invented, without written permission from the Publisher.

For photocopying of material in this volume, please pay a copying fee through the Copyright Clearance Center, Inc., 222 Rosewood Drive, Danvers, MA 01923, USA. In this case permission to photocopy is not required from the publisher.

ISBN $981-238-380-8$ 


\section{Preface}

The theory of operator algebras is generally considered over the field of complex numbers and in the complex Hilbert spaces. So it is a natural and interesting problem: How is in the field of real numbers? Which results are still true in the real case? Which results don't hold in the real case? And which results are needed to change some respects and forms?

Up to now, the theory of operator algebras over the field of real numbers seems not to be introduced systematically and sufficiently. The material in $[17,26]$ seems few and not enough. Moreover, we can also find some respects of the real case in some papers (see References).

Similarly to the complex case, a real operator algebra, precisely speaking, is a $*$ algebra consisting of bounded (real) linear operators on a real Hilbert space, i.e., a * subalgebra of $B(H)$, where $B(H)$ is the collection of all bounded (real) linear operators on a real Hilbert space $H$, and $*$ is the adjoint of operators. Since it is an infinite dimensional object (generally, $H$ is infinite dimensional), so for studying it we must ask that it is closed under some topology. Similarly to the complex case, we find that the closures of real operator algebras with respect to usual locally convex linear topologies in $B(H)$ are just two classes: weak closure and uniform closure. Hence, we need mainly to study the weakly closed real operator algebras (real Von Neumann algebras or real $W^{*}$-algebras) and the uniformly closed real operator algebras (real $C^{*}$-algebras).

The aim of this book is to set up the fundamentals of real operator algebras and to give a systematic discussion for real operator algebras. There are the systematic treatments on complex Banach algebras and complex operator algebras in [1] and [26] respectively. In some sense, this book is a real analogue of [1] and [26] on Banach algebras and operator algebras. Moreover, we shall put other results on real operator algebras into this book 
as more as possible, and give a uniform treatment.

Since our treatment is from beginning (real Banach and Hilbert spaces, real Banach algebras, real Banach $*$ algebras, real $C^{*}$-algebras and $W^{*}-$ algebras, and etc.), and some basic facts are given, we can get some results on real operator algebras easily. However, for the aim of systematic discussion, many results in this book seem to be trivial (i.e., to be a simple movement from the complex case). In this case, we shall just give the statements, and not give their proofs. Readers can find the similar proofs from $[1]$ and [26].

Generally, there are two methods for proving the results on real operator algebras: To move and to change the proofs of the complex case into the real case; and first to go to the complexification and then to go back to the real case. Sometimes just one method is available and another method is not available. Sometimes we must use these two methods simultaneously.

In this book, we shall describe the differences between the complex case and the real case. Moreover, since $A \cong(B,-)$ (real $C^{*}$-algebra, see Chapter 5) and $M \cong(N,-)$ (real $W^{*}$-algebra, see Chapter 6$)$, we shall stress the bar "-" operation throughout this book.

This book is also an introduction for real operator algebras, written in a self-contained manner. For reading it, you just have the general knowledge of Banach algebras and operator algebras, e.g., [1] and [26]. Moreover, we shall use the results in [1] and [26] freely, and not give their proofs.

This book consists of 10 Chapters.

Chapter 1 is the preliminaries. Section 1.1 discusses the complexifications of real Banach spaces and real Hilbert spaces. In particular, we let $\|\xi+i \eta\|=\|\xi-i \eta\|(\forall \xi, \eta)$, i.e., the bar "-" operation is an isometry. Then we can get Propositions 1.1.4 and 1.1.5, and it is important for this book. Section 1.2 is the spectral decomposition in real Hilbert spaces. For (real) normal operators, we need use spectral pair. And for (real) self-adjoint operators, the spectral decomposition theorem is the same as in the complex case.

Chapter 2 contains the complexifications of real Banach algebras, spectrum, divisible real Banach algebras, radical , Arens products, abelian case, and etc. The complexification of a real Banach algebra can be chosen to be a complex Banach algebra, and the bar "-" operation is still isometric. The spectrum of an element must be defined in the complexification, and it is symmetric under the complex conjugation. Proposition 2.4.6 gives a basic fact $(\sigma(x) \cap \mathbb{R}=\{0\}, \forall x \in R(A))$, and we shall use it later. About Arens products, we have Proposition 2.6.4 and etc. Then the regularity of 
real $C^{*}$-algebras can be obtained easily in Chapter 5. Section 2.7 is the Gelfand theory for abelian real Banach algebras. In particular, we give a systematic discussion for the general case (with or without identity).

Chapter 3 is real Banach $*$ algebras. Lemma 3.1.3 gives a basic fact $\left([U(A)] \supset A_{K}\right)$. About abelian case, Theorem 3.2.3 is similar to the complex case, but we must put the hermitian condition. Sections 3.3, 3.4 and 3.5 are GNS construction, $*$ representations, and $*$ radical. Section 3.6 discusses symmetric real Banach $*$ algebras. In particular, the right form of Pták's theory in the real case is given.

Chapter 4 is the fundamentals of real Von Neumann algebras. It is a movement of [26, Chapter 1]. Of course, there are many differences between the complex case and the real case, for examples, $[\overline{P(M)}]=M_{H}(\subsetneq$ $M$ generally), $[U(M)] \subsetneq M$ ( generally, but $[\overline{U(M)}]=M$ ), and etc. Proposition 4.3.3 $\left(M_{c *}=M_{*} \dot{+} i M_{*}\right)$ seems interesting and useful. Moreover, the important Von Neumann's double commutation theorem and Kaplansky's density theorem and etc. are still true in the real case.

Chapter 5 is the fundamentals of real $C^{*}$-algebras. We use the complexification to define real $C^{*}$-algebras, and it is equivalent to the definition in $[3,17]$. In section 5.3 , although $n$-transitivity $(n \geq 2)$ is not true in the real case generally, but we can still prove that 1-transitivity holds and a topologically irreducible $*$ representation is also algebraically irreducible for a real $C^{*}$-algebra. In section 5.5 , we point out that any real $C^{*}$-algebra is regular, and the Arens product in its bidual is the multiplication of operators indeed. The uniqueness of $*$ operation in any real $C^{*}$-algebra is obtained in section 5.6. Section 5.7 is the structure theorem of finite -dimensional real $C^{*}$-algebras, and a method of proof in the theory of operator algebras is given. Section 5.8 is the enveloping real $C^{*}$-algebra of a hermitian real Banach $*$ algebra, and it is a continuity of section 3.6.

Chapter 6 is real $W^{*}$-algebras. It is the abstraction of real Von Neumann algebras, and similarly to Chapter 5 we use the complexification to define real $W^{*}$-algebras. There is also an equivalent definition similar to Sakai's theorem . Section 6.3 discusses abelian real $W^{*}$-algebras and theorem 6.3.6 seems very interesting. Unitaries and partial isometries in real $W^{*}$-algebras are studied in section 6.4.

Gelfand -Naimark conjecture is very famous, and it is a basic problem for the theory of $C^{*}$-algebras. Its real analogue is studied in Chapter 7. Similarly to complex case, we have an affirmative answer for GelfandNaimark conjecture in the real case. These results seem very interesting, and in particular, Theorem 7.2.4 is remarkable. Moreover, the technique of 
"sin" and "cos" is introduced here. Of course, compare with the complex case, there are many open questions.

Chapter 8 discusses the classification of real $W^{*}$-algebras. It is a real analogue of Von Neumann - Murray theory. About first classification (dimension theory), we can study it with the complexification. But for second classification, the real case is more complicated. We have semiabelian projections, semi-discrete real $W^{*}$-algebras, semi-continuous real $W^{*}$-algebras and etc. Here, results are very few, and there are many open questions.

Chapter 9 is a real analogue of reduction theory. The proofs of many results seem to be simple, and the important point is the systematic creation of concepts and notations. In particular, Theorem 9.4.2 seems very interesting compare with the complex case.

Chapter 10 is an introduction for (AF) real $C^{*}$-algebras. An equivalent analytic definition is proved, and it is more complicated than the complex case since finite -dimensional real $C^{*}$-algebras have complicated structure. Moreover, more results on (AF) real $C^{*}$-algebras (e.g., Bratteli diagrams, $K$-theory and etc.) can be found from References.

Moreover, a real $C^{*}$-algebra (or a real von Neumann algebra) can be regarded as a complex $C^{*}$-algebra (or complex von Neumann algebra) with an appropriate conjugation (just look at the complexification) or an appropriate * anti-automorphism. In general, we may have two distinct real $C^{*}$-algebras (or real von Neumann algebras) whose complexifications are the same. An easy example is the real quaternion ring $\mathbb{H}$ and $M_{2}(\mathbb{R})$. They are all non-commutative 4-dimensional real $C^{*}$-algebras (and real von Neumann algebras), but their complexification should be the unique 4-dimensional complex $C^{*}$-algebra (and complex von Neumannn algebra) $M_{2}(\mathbb{C})$ (see Chapter 10). There is a number of research work of the $*$ antiautomorphisms on complex von Neumann algebras or complex $C^{*}$-algebras by E. Stormer, P. J. Stacey, T. Giordano and etc. ([14, 56-59], also see some references in H. Schroder's book [60]). In particular, E. Stormer proved the analogue of Connes' result that a type $I I_{1}$-factor is injective iff it is hyperfinite, for real von Neumannn algebras ([58]): A complex operator algebra can be expressed as the complexification of some real operator algebra if and only if it has a * anti-automorphism. It is still a (very interesting) open question if every complex operator algebra has a $*$ anti-automorphism, i.e. it is an open question if every complex operator algebra is a complexification of some real one. Though some special cases are known (see Stormer's paper). 
Finally, the author acknowledges gratefully the supports of the NSF of China and his home Institute. And the author is also very grateful to Professor Zhong-Jin Ruan for his recommendation.

Bingren $L i$ 
This page is intentionally left blank 


\section{Contents}

Preface $\quad$ v

1. Real Banach and Hilbert Spaces 1

1.1 Complexification of real Banach and Hilbert spaces . . . . . 1

1.2 Spectral decomposition theorem in real Hilbert spaces . . . 9

2. Real Banach Algebras $\quad 15$

2.1 Definition and complexification . . . . . . . . . 15

2.2 Divisible real Banach algebras . . . . . . . . . . 20

2.3 The topological group of invertible elements and its principal

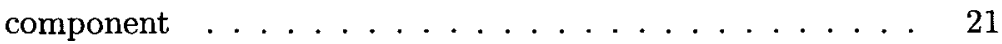

2.4 Radical . . . . . . . . . . . . . . . 22

2.5 Functional calculus . . . . . . . . . . . . . 25

2.6 Arens products . . . . . . . . . . . . . . 26

2.7 Abelian real Banach algebras . . . . . . . . . 28

3. Real Banach * Algebras $\quad 37$

3.1 Some basic lemmas . . . . . . . . . . . . . . . . . 37

3.2 Abelian real Banach $*$ algebras . . . . . . . . . . 40

3.3 Positive linear functionals and GNS construction . . . . . 42

$3.4 *$ Representations and topologically irreducible $*$ representations . . . . . . . . . . . . . 45

$3.5 *$ Radical . . . . . . . . . . . . . . 48

3.6 Symmetric real Banach $*$ algebras $\ldots \ldots \ldots \ldots . \ldots 51$

4. Fundamentals of Real Von Neumann Algebras 59 
4.1 Banach spaces of operators on a real Hilbert space . . . . 59

4.2 Locally convex topologies in $B(H) \ldots \ldots \ldots$. . . . 61

4.3 Von Neumann's double commutation theorem . . . . . . 63

4.4 Kaplansky's density theorem, tensor product commutation theorem, and comparison of projections . . . . . . 68

4.5 Positive linear functionals $\ldots \ldots \ldots \ldots \ldots . \ldots . \ldots 72$

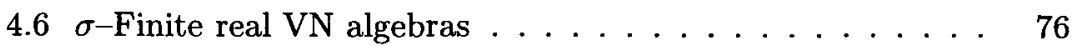

5. Fundamentals of Real $C^{*}$-Algebras $\quad 77$

5.1 Definition and basic properties . . . . . . . . . 77

5.2 Positive functionals and equivalent definition of real $C^{*}$ algebras . . . . . . . . . . . . . 83

5.3 Pure real states, their left kernels, and irreducible $*$ repre-

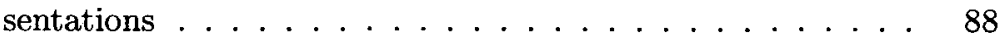

5.4 Ideals, quotient algebras and extreme points . . . . . . . 98

5.5 The bidual of a real $C^{*}$-algebra $\ldots \ldots \ldots \ldots \ldots$

5.6 The uniqueness of $*$ operation . . . . . . . . . . . 104

5.7 Finite-dimensional real $C^{*}$-algebras . . . . . . . . 108

5.8 The enveloping real $C^{*}$-algebra of a hermitian real Banach * algebra . . . . . . . . . . . . 110

$5.9 *$ Representations of abelian real $C^{*}$-algebras $\ldots \ldots \ldots 120$

6. Real $W^{*}$-Algebras 123

6.1 Definition and basic properties . . . . . . . . . . 123

6.2 Normal linear functionals and singular linear functionals . . 130

6.3 Abelian real $W^{*}$-algebras . . . . . . . . . . . . . 132

6.4 Unitaries and partial isometries . . . . . . . . . . . 141

7. Gelfand-Naimark Conjecture in the Real Case 147

7.1 Real $C^{*}$-equivalent algebras . . . . . . . . . . . 147

7.2 The closed unit ball of a unital real $C^{*}$-algebra . . . . . 152

7.3 Gelfand-Naimark conjecture in the real case . . . . . . . . 161

8. Classification of Real $W^{*}$-Algebras $\quad 167$

8.1 Classification of real $W^{*}$-algebras $\ldots \ldots \ldots \ldots 167$

8.2 Finite real $W^{*}$-algebras $\ldots \ldots \ldots \ldots \ldots \ldots \ldots$

8.3 Properly infinite real $W^{*}$-algebras $\ldots \ldots \ldots \ldots \ldots$

8.4 Semi-finite real $W^{*}$-algebras $\ldots \ldots \ldots \ldots \ldots$ 
8.5 Purely infinite (type III) real $W^{*}$-algebras . . . . . . . 183

8.6 Properties on other classes of real $W^{*}$-algebras . . . . . . 184

8.7 Real factors and tensor products $\ldots \ldots \ldots \ldots$

9. Real Reduction Theory 191

9.1 Real measurable fields of Hilbert spaces . . . . . . . . 191

9.2 Real measurable fields of operators . . . . . . . . . . 197

9.3 Real measurable fields of VN algebras . . . . . . . . 200

9.4 Real reduction theory . . . . . . . . . . . . 205

10. (AF) Real $C^{*}$-Algebras 211

10.1 Standard matrix unit $\ldots \ldots \ldots \ldots . \ldots . \ldots 211$

10.2 Technical lemmas . . . . . . . . . . . . . . 218

10.3 Definition and basic properties $\ldots \ldots \ldots \ldots 228$

$\begin{array}{ll}\text { Bibliography } & 233\end{array}$

Notation Index $\quad 237$

Index 239 